\title{
Six-month adherence to Statin use and subsequent risk of major adverse cardiovascular events (MACE) in patients discharged with acute coronary syndromes
}

Gaoqiang Xie ${ }^{1,2}$, Yihong Sun ${ }^{3}$, Phyo Kyaw Myint ${ }^{4}$, Anushka Patel ${ }^{5}$, Xingzi Yang ${ }^{1}$, Min Li ${ }^{6}$, Xian Li ${ }^{7}$, Tao Wu ${ }^{7}$, Shenshen $\mathrm{Li}^{7}$, Runlin $\mathrm{Gao}^{8}$ and Yangfeng $\mathrm{Wu}^{1,2,6,7^{*}}$

\begin{abstract}
Background: The evidence of adherence to statin decreasing risk of major adverse cardiovascular events (MACEs) is still lack among patients discharged with acute coronary syndrome (ACS).

Our objective is to determine the relationship between six-month adherence to statins and subsequent risk of MACEs in patients discharged with ACS.

Methods: Using two prospective registry cohorts (CPACS-1 and -2), we analyzed data from 12,516 consecutive patients with ACS who were prescribed statin at hospital discharge and survived beyond 6 months without recurrent myocardial infarction (MI) or stroke. Adherence to statin was defined as good (using statin at discharge and 6 months without declined dosage) and poor adherence groups (using statin at discharge but declining dosage or stopping at 6 months). We compared the hazard ratios of all-cause mortality and MACE in subsequent 6 months between groups, using Coxregression models, adjusting for multiple potential confounders.
\end{abstract}

Results: Seventy two percent of patients adhered to statin therapy at 6 months. The incident MACE in the poor adherence group was significantly higher than in good adherence group $(2.7 \%$ vs. $1.8 \%, p=0.002)$. Compared with poor adherence group, the good adherence group showed a 27\% lower relative risk of MACE during the 6 month follow up (fully-adjusted hazard ratio $(H R)=0.73 ; 95 \% \mathrm{Cl}$ : $0.56-0.97)$. The protective effects of good adherence were similar in groups with different statin dose as well as groups by other baseline clinical characteristics and treatments ( $p>0.05$ for interaction).

Conclusion: Our study highlights the importance of adherence to statin therapy in prevention of MACE and clinicians should aim to achieve higher dosage if tolerable.

Clinical trial registration: CPACS2 was registered on URL: http://www.anzctr.org.au/default.aspx and unique identifier is ACTRN12609000491268. CPACS1 was not a clinical trial and thus not registered.

Keywords: Adherence, Statin, Major adverse cardiovascular events (MACEs), Acute coronary syndrome (ACS), Cohort

\footnotetext{
* Correspondence: wuyf@bjmu.edu.cn

${ }^{1}$ Peking University Clinical Research Institute, Beijing, China

${ }^{2}$ Key Laboratory of Molecular Cardiovascular Sciences, Ministry of Education,

Beijing, China

Full list of author information is available at the end of the article
} 


\section{Background}

It was estimated that 8.1 million Chinese were affected by coronary heart disease (CHD) in 2010 [1]. The CHD events are set to rise with projected increase by $69 \%$ between 2010 and 2030 in China [2]. Acute coronary syndrome (ACS) is the most serious presentation of CHD. Statins are the main stay of treatment in ACS both acutely and for secondary prevention. Long term use of statins reduces the subsequent risk of major adverse cardiovascular events (MACE) including mortality [3-6] when used in accordance with international clinical practice guidelines including Chinese guidelines [7-11].

Despite the strong evidence of protective effect of statins on subsequent cardiovascular risk, the uptake of statin therapy in China was modest after ACS; recent studies have indicated $\sim 80 \%$ statin prescription at discharge which is compounded by subsequent attrition to adherence rates of less than $60 \%$ by 12 months of follow-up [12, 13].

Medication adherence is defined as the extent to which a patient takes medications as prescribed by their healthcare providers [14]. Poor adherence reduces the effectiveness of essential medications and has been highlighted as a significant obstacle in achieving good patient outcomes [14]. The benefit of adherence to statin on MACE has been confirmed in the primary prevention of coronary artery disease among healthy populations $[15,16]$. However, studies on its effects in secondary prevention are still limited especially in patients discharged with ACS [17-19]. It is also unknown whether the effects are independent of good adherence to other evidence-based drugs therapy or not in these patients. Moreover, in Chinese ACS patients, about $67.1 \%$ did not have known dyslipidaemia [20] and about $42.8 \%$ used low-dose statin $(<20 \mathrm{mg}$ atorvastatin or equivalent) [13]. It is important to quantify the benefit of statin adherence in Chinese ACS patients with poor adherence, to provide incentive for greater adherence to statins for secondary prevention.

We therefore set out to determine the relationship between six-month self-reported adherence to statin use and subsequent risk of major adverse cardiovascular events (MACEs) in Chinese patients with acute coronary syndrome (ACS). We examined the relationship between six-month adherence to statin post ACS and MACE in the following 6 months among 12,516 ACS patients who had been prescribed to use statin at hospital discharge. We also aimed to assess if the relationship was independent of good adherence to other evidence-based beneficial drugs, and was consistent in groups with different statin dose as well as groups by other baseline clinical characteristics and treatments.

\section{Methods}

\section{Study population}

The study sample was drawn from two multicenter prospective registry studies of ACS. They are Clinical
Pathways for Acute Coronary Syndromes in China (CPACS)-1 and-2 which were carried out between 2004 and 2005 and 2007-2009, respectively. The designs and previous findings of these registries have been reported elsewhere [12, 20-22]. The two studies designed by the same study group ensured that data on key variables such as statin usages (yes/no), death, MI, and stroke were collected with the same methods and definitions, providing the foundation to merge them for the current study. A total of 18,038 hospitalised patients with ACS were enrolled. These patients were then followed up at 6 and 12 months after discharge. In the present study, we analyzed 12,516 ACS patients who had been prescribed statins at discharge, survived to 6 months post-discharge without recurrent MI or stroke. These patients were then followed for further 6 months. The study schema is presented in Fig. 1.

\section{Statin data collection}

Data on statin prescription at discharge and at 6 month after discharge were collected for each patient using standardized case report forms by trained staff in both cohorts. The commercial name, and period of using statin were collected. We defined the "good adherence" group as those using statin without decline of dosage between discharge and 6 months post discharge ( $n=9019)$, and the "poor adherence" group as those using statin at discharge but with either cessation or dosage decline at 6 months post discharge $(n=3497)$. Dosage of statin was only collected in CPACS-2. In order to examine the interaction of statin dose on the relationship of statin adherence with MACE, we performed in subgroups by statin dose (5-10 $\mathrm{mg} / \mathrm{d}$ and $>=20 \mathrm{mg} / \mathrm{d}$ equivalent dose of atorvastatin) using data from CPACS-2. For our analysis, the dosage of different

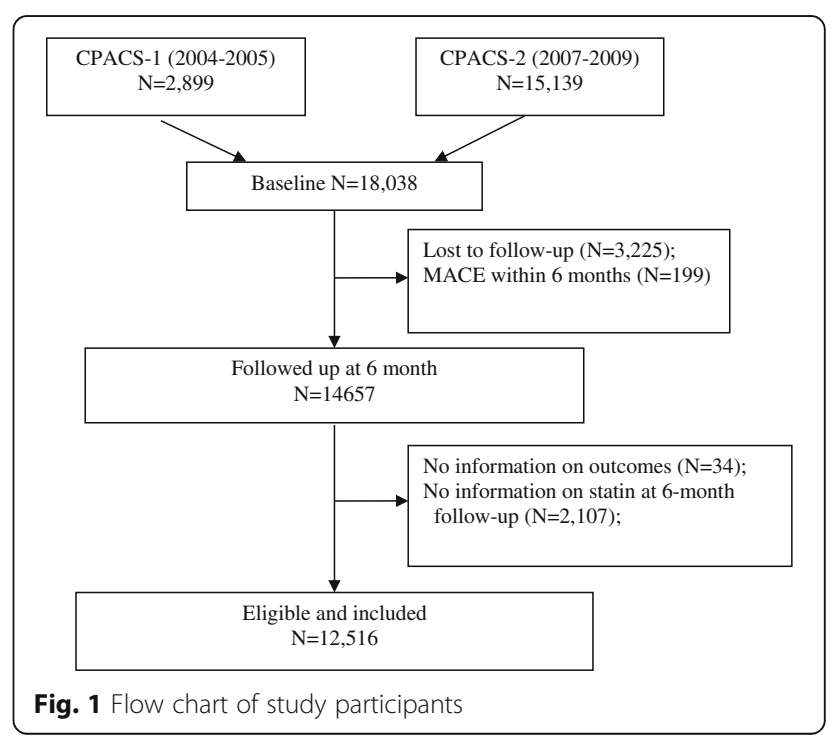


statins was converted to the equivalent dosage of atorvastatin according to Chinese Guidelines on Prevention and Treatment of Dyslipidemia in Adults in $2007^{7}$ (Additional file 1: Table S1).

\section{Outcomes ascertainment}

Data on outcomes including all-cause mortality, incident myocardial infarction (including fatal and non-fatal), and stroke (including fatal and non-fatal) were collected by two follow-up surveys (by clinic visit or telephone) at 6 and 12 months after discharge [12]. Major adverse cardiovascular event (MACE) was defined as composite end point of all-cause mortality, myocardial infarction, or stroke. All myocardial infarction and stroke events were diagnosed in hospitals by their physicians. In the present study, the outcome of interest was MACE occurring during 7-12 months after discharge and therefore, deaths, stroke or MI occurring within 6 months after discharge were excluded from the study.

\section{Other data collection}

Other data collected during hospitalization included sociodemographic characteristics, medical history, features of the presenting condition, electrocardiographic (ECG) and plasma biomarker findings, as well as treatment received at the baseline and during hospitalization. Other data collected at discharge and 6-month followup included other prescribed medicines (aspirin, clopidogrel, beta-blocker, and angiotensin-converting enzyme inhibitor/angiotensin receptor blocker), medical history, presenting features, and diagnosis at discharge. These variables were analyzed as potential confounders in the present study.

\section{Statistical analyses}

For descriptive purpose, means and standard deviations were calculated to present continuous variables and were compared by t-tests between poor and good adherence groups. Categorical variables were presented as number (percentage) and differences between groups were tested by Chi-squared analysis. Cox proportional hazards regression models (unadjusted, age- and sex- adjusted, and multiple co-variables-adjusted) were constructed to calculate hazard ratios (HR) and corresponding 95\% confidence intervals $(95 \% \mathrm{CI})$ for MACE occurring within 712 month post discharge. The co-variables that were adjusted in the models were selected based on the association metrics analyses and on the current professional knowledge. These co-variables included studies (CPACS$1,-2)$, subtypes of ACS, hospital level, health insurance, social economic status, clinical characteristics, concomitant medicines and reperfusion therapies during hospitalization, and adherence to other evidence-based secondary prevention treatments for ACS in the first
6 months, in addition to age and sex. All models were then also constructed for all-cause mortality, incident MI and stroke, separately.

Subgroup analyses were also performed with the Cox models in populations defined by age $(<$ or $>=65$ years $)$, sex, type of ACS, presence of hypertension or, diabetes at baseline, smoking level, hospital type, baseline cardiovascular risk factors, coronary revascularization during hospital admission, dose of statin, adherence to other medications.

All $p$ values reported were two sided, and a $p$ value of $<0.05$ was considered significant. All analyses were performed using SAS 9.4 (SAS Institute Inc., Cary, NC, USA).

\section{Results}

Baseline characteristics

A total of the 12,516 patients (9019 men and 3497 women) were eligible to be included in this report (Fig. 1). The mean age (SD) of the cohort was 63.3 (11.5) years at the time of hospitalization. The rate of good adherence was $72 \%(9019 / 12516)$ at 6 months after discharge. Compared with the poor adherence group, the good adherence group had a higher proportion of men, and were more likely to have a higher education level, to be treated in tertiary hospital, to have health insurance, to have diabetes mellitus, dyslipidemia, and taking statins before hospitalization. During hospitalization, the good adherence group were more likely to have ECG monitoring, and to receive PCI/CABG. They also were more likely to had good adherence to other medications at 6 months post discharge, including aspirin, clopidogrel, beta-blocker, and angiotensin-converting enzyme inhibitor/angiotensin receptor blocker (Table 1). The proportions of participants with $5,10,20$, and $\geq 40 \mathrm{mg} / \mathrm{d}$ atorvastatin equivalent dose at discharge were $21,33,44$, and $2 \%$, respectively. The dosage levels were significantly higher in patients who were managed in tertiary hospitals compared to level-2 hospitals $(p<0.001)$ (Fig. 2).

\section{Adherence to statin and MACE}

Among 12,516 patients with event-free survival to 6 months follow up, a total of 259 MACE occurred during the subsequent 6 months. The crude incidence rate of MACE events was statistically significantly lower in the good adherence group compared to the poor adherence group. After adjustment for co-variables, the association was attenuated, but remained statistically significant (Table 2).

\section{Adherence to statin and components of MACEs}

There were a total of 146, 96 and 49 patients resulted in death, myocardial infarction and stroke during follow up. The crude incidence rate of almost components of MACE was statistically significantly lower in the good adherence group compared to the poor adherence group, but after adjustment for co-variables, the associations were attenuated and not statistically significant (Table 2). 
Table 1 Baseline clinical characteristics according to the statin use adherence at six months after discharge

\begin{tabular}{|c|c|c|c|}
\hline Baseline variables & $\begin{array}{l}\text { Good adherence } \\
(n=9019)\end{array}$ & $\begin{array}{l}\text { Poor adherence } \\
(n=3497)\end{array}$ & $P$-value \\
\hline \multicolumn{4}{|l|}{ Studies, n(\%) } \\
\hline CPACS-1 & 1415(15.7) & $512(14.6)$ & \multirow[t]{2}{*}{0.145} \\
\hline CPACS-2 & $7604(84.3)$ & $2985(85.4)$ & \\
\hline Male, n(\%) & $6380(70.7)$ & $2390(68.3)$ & 0.009 \\
\hline Age,mean(SD) & $63.33(11.5)$ & 63.34(11.8) & 0.974 \\
\hline High School or higher, n(\%) & $3444(38.2)$ & 1252(35.8) & 0.013 \\
\hline \multicolumn{4}{|l|}{ Occupations, n(\%) } \\
\hline Employed & $4605(51.1)$ & $1767(50.5)$ & \multirow[t]{3}{*}{0.844} \\
\hline Not-employed & $4214(46.7)$ & 1654(47.3) & \\
\hline Unknown & $200(2.2)$ & $76(2.2)$ & \\
\hline Tertiary hospital, n(\%) & $5781(64.1)$ & 1987(56.8) & $<0.001$ \\
\hline Having health insurance, n(\%) & 7308(81.0) & $2700(77.2)$ & $<0.001$ \\
\hline Smoking, n(\%) & $2748(30.5)$ & 1096(31.3) & 0.343 \\
\hline \multicolumn{4}{|l|}{ Subtype of ACS, n(\%) } \\
\hline STEMI & $3530(39.1)$ & 1347(38.5) & \multirow[t]{3}{*}{0.104} \\
\hline Non-STEMI & 1206(13.4) & $427(12.2)$ & \\
\hline Unstable angina & $4283(47.5)$ & 1723(49.3) & \\
\hline \multicolumn{4}{|l|}{ CVD risk factors, $\mathrm{n}(\%)$} \\
\hline Hypertension & $6354(70.5)$ & $2402(68.7)$ & 0.053 \\
\hline Diabetes Mellitus & 1892(21.0) & $655(18.7)$ & 0.005 \\
\hline Dyslipidemia $^{*}$ & 4807(53.3) & 1734(49.6) & $<0.001$ \\
\hline Smoking & $2748(30.5)$ & 1096(31.3) & 0.343 \\
\hline Obesity & $897(10.0)$ & $321(9.2)$ & 0.194 \\
\hline Family history of early CHD, n(\%) & 414(4.6) & $171(4.9)$ & 0.476 \\
\hline Higher heart rate(> = 100/min),n(\%) & $657(7.3)$ & $277(7.9)$ & 0.224 \\
\hline Continuous ECG monitoring, n(\%) & $6487(71.9)$ & 2436(69.7) & 0.012 \\
\hline \multicolumn{4}{|l|}{ Treatments during hospitalization, n(\%) } \\
\hline $\mathrm{PCl} / \mathrm{CABG}$ & $4746(52.6)$ & 1334(38.2) & $<0.001$ \\
\hline Thrombolysis & $608(6.7)$ & $318(9.1)$ & $<0.001$ \\
\hline Statin before hospitalization & 1681(18.6) & $513(14.7)$ & $<0.001$ \\
\hline $\mathrm{PCl} / \mathrm{CABG}$ in $0-6$ months, $\mathrm{n}(\%)$ & $308(3.4)$ & 131(3.8) & 0.3663 \\
\hline \multicolumn{4}{|l|}{ Adherence of other medications in $0-6$ months, $\mathrm{n}(\%)$} \\
\hline Aspirin & $8573(95.1)$ & $2742(78.4)$ & $<0.001$ \\
\hline Clopidogrel & $5962(66.1)$ & $1414(40.4)$ & $<0.001$ \\
\hline Beta-blocker & $6602(73.2)$ & 1772(50.7) & $<0.001$ \\
\hline Angiotensin-converting enzyme inhibitor/Angiotensin receptor blocker & 6312(70.0) & 1493(42.7) & $<0.001$ \\
\hline
\end{tabular}

*Dyslipidemia was defined as having a history of dyslipidemia or total serum cholesterol $>=240 \mathrm{mg} / \mathrm{dL}$, or $\mathrm{LDL}-\mathrm{c}>=160 \mathrm{mg} / \mathrm{dL}$, or triglyceride $>=200 \mathrm{mg} / \mathrm{dL}$, or $\mathrm{HDL}-\mathrm{c}<40 \mathrm{mg} / \mathrm{dL}$ according to the guideline of China

\section{Sub-group analyses}

All associations of adherence to statin with MACE were consistent between study cohorts and pre-defined subgroups of baseline characteristics including the sub-types of ACS (Fig. 3). There were not significant interactions between adherence to statin and other main exposure factors.

\section{Discussion}

Our results demonstrate that good adherence to statin in the first 6 months after discharge is associated with subsequent lower incidence of MACE (including allcause mortality, MI, and stroke) in a large unselected Chinese ACS patient population. This relationship was 


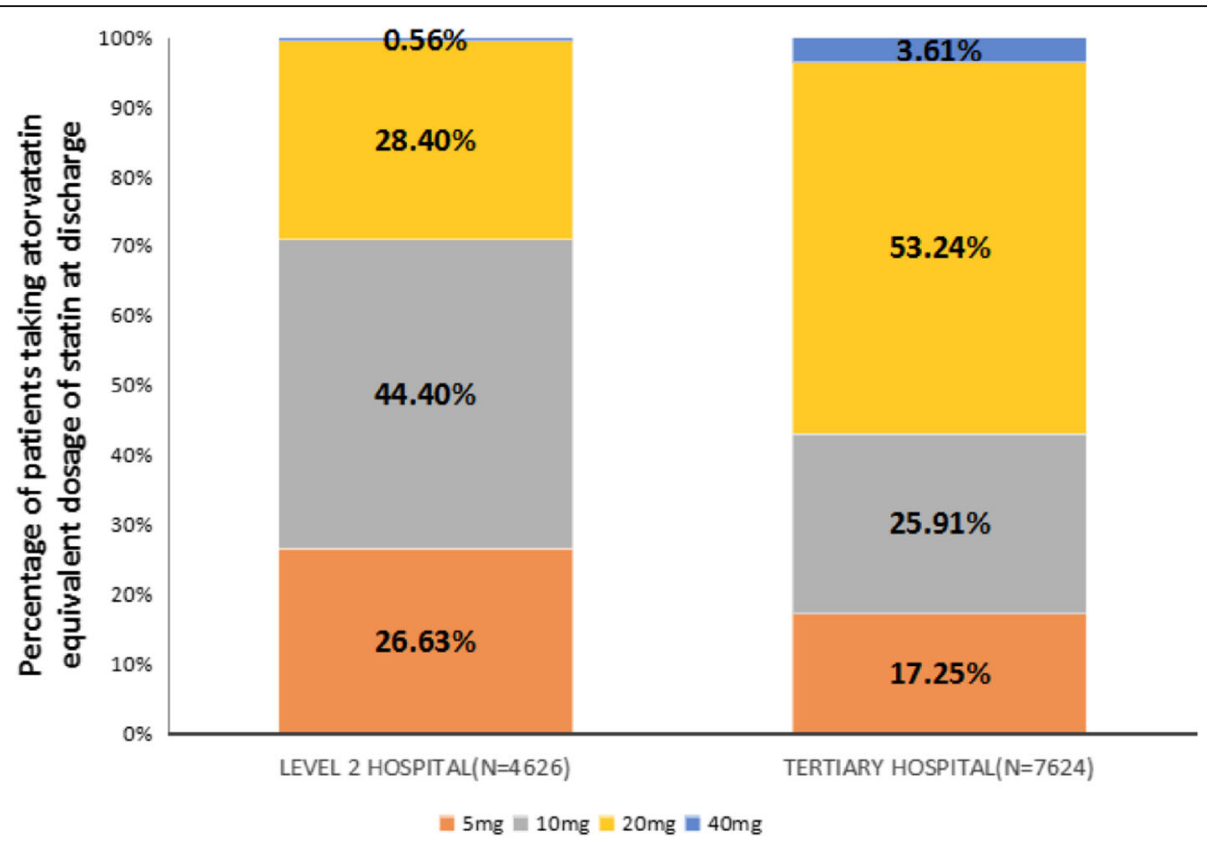

Fig. 2 The proportion of patients prescribed different statin dosage in tertiary hospitals and level 2 hospitals $(p<0.001)$

independent of other treatments which have been shown to be associated with outcomes in ACS such as receipt of coronary interventions, and adherence to antiplatelets, aspirin, beta-blocker, and angiotensinconverting enzyme inhibitor/angiotensin receptor blocker, as well as other known personal prognostic factors such as age, sex, education level, and cardiovascular risk factors and other hospital level characteristics. In addition, the effect of adherence was not modified by the characteristics of patients such as dose of statins, subtypes of ACS, gender, age, social economic status, etc.

It has been well established that good statin adherence is associated with reduced the risk of MACE and allcause mortality in the primary prevention of coronary artery disease among healthy populations $[15,16]$. The benefits associated with statin adherence in secondary prevention have only been reported among elderly AMI

Table 2 Clinical Outcomes at 7-to-12 Months between poor and good adherence group

\begin{tabular}{|c|c|c|c|c|c|}
\hline \multirow[t]{2}{*}{ Outcomes } & \multirow{2}{*}{$\begin{array}{l}\text { Adherence } \\
\text { groups }\end{array}$} & \multirow{2}{*}{$\begin{array}{l}\text { Number of } \\
\text { events (\%) }\end{array}$} & \multicolumn{3}{|l|}{$\mathrm{HR}(95 \% \mathrm{Cl})$} \\
\hline & & & Unadjusted & Age-sex-adjusted & Fully-adjusted $^{a}$ \\
\hline \multirow[t]{3}{*}{$\overline{M A C E}$} & Poor adherence & $95(2.72)$ & 1 (ref.) & 1 (ref.) & 1 (ref.) \\
\hline & Good adherence & $164(1.82)$ & $0.66(0.51,0.85)$ & $0.68(0.52,0.87)$ & $0.73(0.56,0.97)$ \\
\hline & $P$ values & 0.002 & 0.001 & 0.002 & 0.030 \\
\hline \multirow[t]{3}{*}{ Total death } & Poor adherence & $56(1.60)$ & 1 (ref.) & 1 (ref.) & 1 (ref.) \\
\hline & Good adherence & $90(1.00)$ & $0.62(0.44,0.86)$ & $0.63(0.45,0.88)$ & $0.71(0.49,1.04)$ \\
\hline & $P$ values & 0.005 & 0.005 & 0.007 & 0.077 \\
\hline \multirow[t]{3}{*}{$\mathrm{Ml}$} & Poor adherence & $30(0.86)$ & 1 (ref.) & 1 (ref.) & 1 (ref.) \\
\hline & Good adherence & $66(0.73)$ & $0.84(0.55,1.3)$ & $0.85(0.55,1.3)$ & $0.88(0.55,1.41)$ \\
\hline & $P$ values & 0.468 & 0.444 & 0.447 & 0.6 \\
\hline \multirow[t]{3}{*}{ Stroke } & Poor adherence & $21(0.60)$ & 1 (ref.) & 1 (ref.) & 1 (ref.) \\
\hline & Good adherence & $28(0.31)$ & $0.51(0.29,0.9)$ & $0.53(0.3,0.93)$ & $0.56(0.3,1.02)$ \\
\hline & $P$ values & 0.020 & 0.02 & 0.026 & 0.059 \\
\hline
\end{tabular}

adjusted for studies (CPACS-1,-2), age, sex, education, occupations, have health insurance, hospital level, cardiovascular risk factors (hypertension, diabetes, dyslipidemia, obesity, smoking, and family history of early CHD), higher heart rate( $>=100 / \mathrm{min}$ ), continuous ECG monitoring, subtype of ACS, thrombolysis, statin before hospitalization, PCI/CABG during hospitalization, PCl/CABG from 0- to 6- month after discharge, and adherence to clopidogrel, aspirin, beta-blocker, and ACEI/ARB 

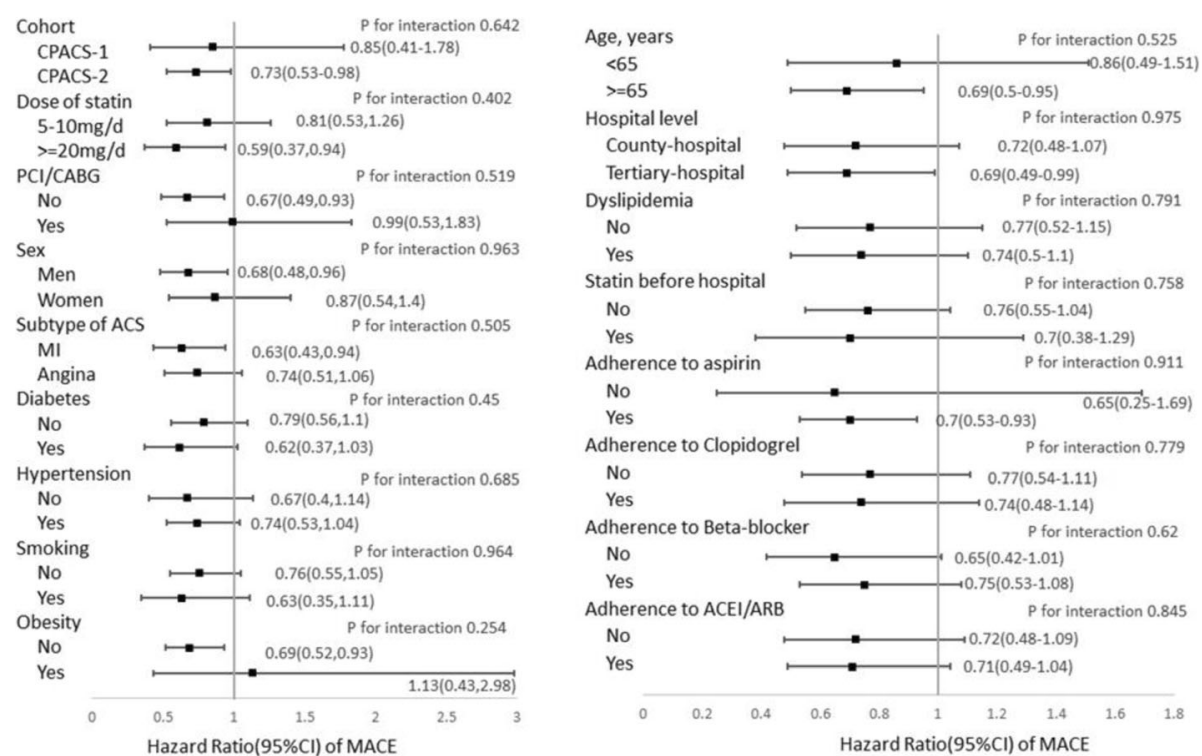

Fig. 3 Hazard ratios of major adverse cardiovascular events (MACEs) for patients with good adherence compared to patients with poor adherence by cohorts (CPACS-1 and -2) and baseline characteristics. HRs were estimated with Cox model with adjustment for all other covariables shown in the fig. $\mathrm{PCl}=$ percutaneous coronary intervention. $\mathrm{CABG}=$ coronary artery bypass graft. $\mathrm{ACEl}=$ angiotensin converter enzyme inhibitor. $\mathrm{ARB}=$ angiotensin receptor blocker

survivors for more than 1 year and three months ${ }^{18}$ and in known CVD (angina, myocardial infarction, heart failure, stroke/transient ischaemic attack and peripheral vascular disease as a whole) patients [19]. Such evidence among patients discharged with ACS is lacking and to the best of our knowledge, the present study is the first one to show good adherence to statin therapy during the first 6 months after discharge was significantly associated with lower risk of MACE in subsequent 6 months among ACS patients. Moreover, we found the relationship was not modified by statin dose, subtype of ACS, PCI/CABG, dyslipidemia, other clinical characteristics, and demographics, and was independent of good adherence to other evidence-based beneficial drugs, including antiplatelets, beta-blocker, and angiotensin-converting enzyme inhibitor/angiotensin receptor blocker at same time. Our findings suggest that adherence to statin is beneficial even in those ACS among whom about 50\% did not have dyslipidemia and $54 \%$ had lower statin dose ( $<20 \mathrm{mg}$ atorvastatin or equivalent).

Our findings highlight the importance to improve adherence to statin in the secondary prevention among ACS patients among Asian populations. We note that the level of good adherence to statin rate was only $72 \%$ at the 6 months after discharge in our study; considering the expected even lower adherence at 12 months as shown in CPACS-1 study [12], it was substantially lower than the adherence reported in Canadians [18]. Therefore, there is clearly room for improvement and ensuring good adherence to statins may translate into substantial clinical benefit with regard to MACE.

Our findings for each of the MACE components showed that the hazard ratio for patients with good adherence vs poor adherence was all less than 1.0 and borderline significant. We consider the results are coherent with the significant findings for the primary study outcome. Our ad-hoc power analysis indicated that the study power is $87 \%$ to detect the observed effect size on the primary outcome, MACE, $78 \%$ on total death, $13 \%$ on $\mathrm{MI}$ and $63 \%$ on stroke, with $\mathrm{a}=0.05$ among 12,500 study participants.

\section{Strengths}

The participants were drawn from CPACS1 and CPACS2 which were well designed studies with high scientific quality standards [12, 20-22]. Firstly, we assessed the relationship of adherence to statin at 6 months after discharge with subsequent clinical outcomes during 7-12 months after excluding those patients with MI or stroke events in the first 6 months. This approach would avoid bias towards a result unfavorable to good adherence because the patients with recurrence of $\mathrm{MI} /$ stroke during the first 6 months were more likely to take statin at 6 month and hence be defined as adhere to statin in our study. Secondly, we controlled all available potential confounders including studies(CPACS-1,-2), age, sex, health insurance, social economic status, clinical characteristics, use statin before hospital, concomitant medicines and reperfusion therapies during hospitalization, and adherence to other 
evidence-based secondary prevention treatments for ACS in the first 6 months.

\section{Limitations}

There are some limitations. First, we only defined good adherence as using statin without dosage reduction at 6 months, and poor adherence as using statin at discharge but dosage declined or discontinued usage at 6 months. Compared with common definition using the proportion of days covered (PDC) $>=80 \%$ as good adherence, and $40-79 \%$ as poor adherence [23], we possibly misclassified some patients and thus under-estimated the strength of the associations observed. Second, as an observational study, we cannot assert causality due to the unmeasured and unknown confounding factors. Third, our data on adherence was self-reported, though a sample of study participants were randomly selected and central calls were made to confirm the quality of follow up data. Fourth, there was no independent adjudications of events in our study which make the diagnoses of $\mathrm{MI} /$ stroke might not be accurate. Finally, we only assessed the adherence at a single time point at relatively short interval of six-month post-discharge. Compared with longer-term adherence, the short-term adherence may have smaller magnitude of benefit as statins benefit has been more commonly acknowledged over at least 12-18 month therapy.

\section{Conclusion}

In summary, this study found that ACS patients with good adherence to statin in the first 6 months after discharge had significantly lower risk of subsequent MACE in the following 7 to 12 months. Our study highlights the importance of adherence to cholesterol lowering therapy in prevention of MACE. Whilst the results are broadly similar to previous reports in Western populations, our study provide strong incentive with regard to relevance of statin adherence in reduction in MACE in the Chinese ACS patients.

\section{Additional file}

Additional file 1: Table S1. Comparative Dose Efficacy of Statins on lipids (DOCX $15 \mathrm{~kb}$ )

\section{Abbreviations \\ ACEl: Angiotensin converter enzyme inhibitor; ACS: Acute coronary syndrome; ARB: Angiotensin receptor blocker; CABG: Coronary artery bypass graft; Cl: Confidence interval; HR: Hazard ratio; MACEs: Major adverse cardiovascular events; MI: Myocardial infarction; PCI: Percutaneous coronary intervention; STEMI: ST segment elevation myocardial infarction}

\section{Funding}

CPACS-1 was funded by unrestricted educational grants from Guidant and Sanofi-Aventis, and grants from The Royal Australasian College of Physicians. AP is supported by an Australian National Heart Foundation Career Development Award. CPACS-2 was funded by an unrestricted grant from SanofiAventis China. The George Institute for Global Health at Peking University Health Science Center sponsored the study and owns the data. Data analyses and reports were supported by Beijing Science and Technology Key Research Plan (D151100002215001). However, the authors are solely responsible for the design, analyses, the drafting and editing of the manuscript, and its final contents.

\section{Availability of data and materials}

The authors declare that the data supporting the findings of this study are available within the article.

\section{Authors' contributions}

GX: concept development, data cleaning analysis, and interpretation, and writing of the manuscript; YS: critical input in interpretation of results and writing of the manuscript; PKM: critical input in interpretation of results and writing of the manuscript; AP: critical input in interpretation of results and writing of the manuscript; $X Y$ : data analyses; ML: review of manuscript; $\mathrm{XL}$ : data analysis plan and review of manuscript; TW: data analysis plan and review of manuscript; SL: data collection and review of manuscript; RG: review of manuscript and critical input in interpretation of results; YW: concept development, critical input in interpretation of results, and review and approval of the manuscript.

\section{Ethics approval and consent to participate}

CPACS-1 and -2 studies were approved by the Ethics Committee at Fuwai Hospital and University of Sydney the studies. A written informed consent was acquired from all participants before recruitment into the study. De-identified data were used for data analysis.

\section{Consent for publication}

Not applicable.

\section{Competing interests}

The authors declare that they have no competing interests.

\section{Publisher's Note}

Springer Nature remains neutral with regard to jurisdictional claims in published maps and institutional affiliations.

\section{Author details}

${ }^{1}$ Peking University Clinical Research Institute, Beijing, China. ${ }^{2}$ Key Laboratory of Molecular Cardiovascular Sciences, Ministry of Education, Beijing, China. ${ }^{3}$ China-Japan Friendship Hospital, Beijing, China. ${ }^{4}$ School of Medicine, Medical Sciences and Nutrition, University of Aberdeen, Aberdeen, UK. ${ }^{5}$ The George Institute for Global Health, University of Sydney, Sydney, Australia.

${ }^{6}$ Department of Epidemiology and Biostatistics, |Peking University School of Public Health, Beijing, China. ${ }^{7}$ The George Institute for Global Health at Peking University Health Science Center, Beijing, China. ${ }^{8}$ The Department of Cardiology, Fuwai Hospital, National Center for Cardiovascular Diseases, Chinese Academy of Medical Sciences, Beijing, China.

Received: 14 May 2017 Accepted: 2 August 2017 Published online: 15 August 2017

\section{References}

1. Wang S, Marquez P, Langenbrunner J. Toward a healthy and harmonious life in china: stemming the rising tide of rnon-communicable diseases. DC: World Bank: Washington; 2011.

2. Moran A, Zhao D, Gu D, Coxson P, Chen CS, Cheng J, Liu J, He J, Goldman $L$. The future impact of population growth and aging on coronary heart disease in china: projections from the coronary heart disease policy modelchina. BMC Public Health. 2008;8:394.

3. Larsen Al, Tomey MI, Mehran R, Nilsen DW, Kirtane AJ, Witzenbichler B, Guagliumi G, Brener SJ, Genereux P, Kornowski R, Dudek D, Gersh BJ, Stone GW. Comparison of outcomes in patients with st-segment elevation myocardial infarction discharged on versus not on statin therapy (from the 
harmonizing outcomes with revascularization and stents in acute myocardial infarction trial). Am J Cardiol. 2014;113:1273-9.

4. Lenderink T, Boersma E, Gitt AK, Zeymer U, Wallentin L, Van de Werf F, Hasdai D, Behar S, Simoons ML. Patients using statin treatment within $24 \mathrm{~h}$ after admission for st-elevation acute coronary syndromes had lower mortality than non-users: a report from the first euro heart survey on acute coronary syndromes. Eur Heart J. 2006;27:1799-804

5. Schwartz GG, Olsson AG, Ezekowitz MD, Ganz P, Oliver MF, Waters D, Zeiher A, Chaitman BR, Leslie S, Stern T. Effects of atorvastatin on early recurrent ischemic events in acute coronary syndromes: the miracl study: a randomized controlled trial. JAMA. 2001;285:1711-8.

6. Stenestrand U, Wallentin L. Early statin treatment following acute myocardial infarction and 1-year survival. JAMA. 2001;285:430-6.

7. Editor committee of chinese guidelines on prevention and treatment of dyslipidemia in adults. Chinese guidelines on prevention and treatment of dyslipidemia in adults in 2007. Chin J Cardiol. 2007;35:390-419.

8. Anderson JL, Adams CD, Antman EM, Bridges CR, Califf RM, Casey DE Jr, Chavey WE 2nd, Fesmire FM, Hochman JS, Levin TN, Lincoff AM, Peterson ED, Theroux P, Wenger NK, Wright RS, Jneid H, Ettinger SM, Ganiats TG, Philippides GJ, Jacobs AK, Halperin JL, Albert NM, Creager MA, DeMets D, Guyton RA, Kushner FG, Ohman EM, Stevenson W, Yancy CW. 2012 accf/aha focused update incorporated into the accf/aha 2007 guidelines for the management of patients with unstable angina/non-st-elevation myocardial infarction: a report of the american college of cardiology foundation/ american heart association task force on practice guidelines. J Am Coll Cardiol. 2013;61:e179-347.

9. Anderson JL, Adams CD, Antman EM, Bridges CR, Califf RM, Casey DE Jr, Chavey WE 2nd, Fesmire FM, Hochman JS, Levin TN, Lincoff AM, Peterson ED, Theroux P, Wenger NK, Wright RS, Smith SC Jr, Jacobs AK, Adams CD, Anderson JL, Antman EM, Halperin JL, Hunt SA, Krumholz HM, Kushner FG, Lytle BW, Nishimura R, Ornato JP, Page RL, Riegel B. Acc/aha 2007 guidelines for the management of patients with unstable angina/non-stelevation myocardial infarction: a report of the american college of cardiology/american heart association task force on practice guidelines (writing committee to revise the 2002 guidelines for the management of patients with unstable angina/non-st-elevation myocardial infarction) developed in collaboration with the american college of emergency physicians, the society for cardiovascular angiography and interventions, and the society of thoracic surgeons endorsed by the american association of cardiovascular and pulmonary rehabilitation and the society for academic emergency medicine. J Am Coll Cardiol. 2007;50:e1-e157.

10. Jneid H, Anderson JL, Wright RS, Adams CD, Bridges CR, Casey DE Jr, Ettinger SM, Fesmire FM, Ganiats TG, Lincoff AM, Peterson ED, Philippides GJ, Theroux P, Wenger NK, Zidar JP, Anderson JL. 2012 accf/aha focused update of the guideline for the management of patients with unstable angina/non-st-elevation myocardial infarction (updating the 2007 guideline and replacing the 2011 focused update): a report of the american college of cardiology foundation/american heart association task force on practice guidelines. Circulation. 2012;126:875-910.

11. Stone NJ, Robinson JG, Lichtenstein AH, Bairey Merz CN, Blum CB, Eckel RH, Goldberg AC, Gordon D, Levy D, Lloyd-Jones DM, McBride P, Schwartz JS, Shero ST, Smith SC Jr, Watson K, PWF W, American College of Cardiology/ American Heart Association Task Force on Practice G. 2013 acc/aha guideline on the treatment of blood cholesterol to reduce atherosclerotic cardiovascular risk in adults: a report of the american college of cardiology/ american heart association task force on practice guidelines. J Am Coll Cardiol. 2014;63:2889-934.

12. Bi Y, Gao R, Patel A, Su S, Gao W, Hu D, Huang D, Kong L, Qi W, Wu Y, Yang $Y$, Turnbull F. Evidence-based medication use among chinese patients with acute coronary syndromes at the time of hospital discharge and 1 year after hospitalization: results from the clinical pathways for acute coronary syndromes in china (cpacs) study. Am Heart J. 2009;157:509-16. e501

13. Zhang L, Li J, Li X, Nasir K, Zhang H, Wu Y, Hu S, Wang Q, Downing NS, Desai NR, Masoudi FA, Spertus JA, Krumholz HM, Jiang L. National assessment of statin therapy in patients hospitalized with acute myocardial infarction: insight from china peace-retrospective ami study, 2001, 2006, 2011. PLoS One. 2016;11:e0150806.

14. Osterberg L, Blaschke T. Adherence to medication. N Engl J Med. 2005;353:487-97.

15. Perreault S, Dragomir A, Blais L, Berard A, Lalonde L, White M, Pilon D. Impact of better adherence to statin agents in the primary prevention of coronary artery disease. Eur J Clin Pharmacol. 2009;65:1013-24.
16. Rannanheimo PK, Tiittanen P, Hartikainen J, Helin-Salmivaara A, Huupponen $\mathrm{R}$, Vahtera J, Korhonen MJ. Impact of statin adherence on cardiovascular morbidity and all-cause mortality in the primary prevention of cardiovascular disease: a population-based cohort study in finland. Value in health : the journal of the International Society for Pharmacoeconomics and Outcomes Research. 2015;18:896-905.

17. Chowdhury R, Khan H, Heydon E, Shroufi A, Fahimi S, Moore C, Stricker B, Mendis S, Hofman A, Mant J, Franco OH. Adherence to cardiovascular therapy: a meta-analysis of prevalence and clinical consequences. Eur Heart J. 2013;34:2940-8

18. Rasmussen JN, Chong A, Alter DA. Relationship between adherence to evidence-based pharmacotherapy and long-term mortality after acute myocardial infarction. JAMA. 2007;297:177-86.

19. Wei L, Fahey T, MacDonald TM. Adherence to statin or aspirin or both in patients with established cardiovascular disease: exploring healthy behaviour vs. drug effects and 10-year follow-up of outcome. Br J Clin Pharmacol. 2008;66:110-6.

20. Gao R, Patel A, Gao W, Hu D, Huang D, Kong L, Qi W, Wu Y, Yang Y, Harris $P$, Algert $C$, Groenestein $P$, Turnbull F. Prospective observational study of acute coronary syndromes in china: practice patterns and outcomes. Heart. 2008;94:554-60.

21. Du X, Gao R, Turnbull F, Wu Y, Rong Y, Lo S, Billot L, Hao Z, Ranasinghe I, ledema R, Kong L, Hu D, Lin S, Shen W, Huang D, Yang Y, Ge J, Han Y, Lv S, Ma A, Gao W, Patel A. Hospital quality improvement initiative for patients with acute coronary syndromes in china: a cluster randomized, controlled trial. Circulation. Cardiovascular quality and outcomes. 2014;7:217-26.

22. Rong Y, Turnbull F, Patel A, Du X, Wu Y, Gao R. Clinical pathways for acute coronary syndromes in china: protocol for a hospital quality improvement initiative. Critical pathways in cardiology. 2010;9:134-9.

23. Chan DC, Shrank WH, Cutler D, Jan S, Fischer MA, Liu J, Avorn J, Solomon D, Brookhart MA, Choudhry NK. Patient, physician, and payment predictors of statin adherence. Med Care. 2010;48:196-202.

\section{Submit your next manuscript to BioMed Central and we will help you at every step:}

- We accept pre-submission inquiries

- Our selector tool helps you to find the most relevant journal

- We provide round the clock customer support

- Convenient online submission

- Thorough peer review

- Inclusion in PubMed and all major indexing services

- Maximum visibility for your research

Submit your manuscript at www.biomedcentral.com/submit
Biomed Central 